hören der Verbreitung des Emphysems erwarten kann. Therapeutisch wurde in meinem Falle gegen dasselbe gar nicht eingeschritten und die Zange nur wegen zu langer Dauer der Austreibungsperiode angelegt, doch konnte sie auch durch das Emphysem indicirt sein. Die Beschreibung der Chirurgen sowie Therapenten zeigt die Zeichnung in Billroth's Chirurgie, welche colossale Ausbreitung z. B. ein Hautemphysem erlangen und zu welchen schweren Störungen des Kreislaufes dasselbe führen kann; aus meinem Falle geht hervor, dass ein ziemlich ausgebreitetes Emphysem im Wochenbette ohne alle Therapie von selbst schwindet. Sollte man während der Geburt ein Auwachsen desselben finden, so müsste jene schon deshalb beschleunigt werden.

\title{
Notiz über den Bau des Fungus umbilicalis.
}

\author{
Von \\ Dr. O. Küstner, \\ Assisten $\downarrow$ am gynäkologischen Institute in Falle.
}

Vor ungefähr einem Jahre untersuchte ich einen Fungus umbilicalis, der mit der Scheere vom Nabel eines dreimonatlichen Kindes abgetragen war, mikroskopisch. Ich erwartete nichts weiter als blosses Granulationsgewebe zu finden, und war nicht wenig überrascht, anstatt dieser einfachsten Structur an dem Tumor einen relativ complicirten Bau zu entdecken: Im Centrum, von der Abtragungsstelle an, straffes faseriges Bindegewebe, nach aussen davon dichtgedrängte Rundzellen (Granulationsgewebe) und in dasselbe eingebettet die ganze Peripherie des Tumors umgebend zahlreiche Drüsen. Dieselben sind röhrenförmig, haben, während die ganze Geschwulst die Grösse einer Erbse besitzt, etwa die Länge von $1 / 6$ ihres Durchmessers, und sind ausgekleidet von oben bis in den Fundus mit schönem Cylinderepithel. Sie stehen dicht eine neben der anderen, und laufen in ziemlich gestreckter Richtung; einige derselben theilen sich nahe dem Fundus gabelig, die meisten jedoch sind einfach. Das Epithel des Tumors, das sich von einer Drüsenmundung zur anderen begiebt, ist einschichtig, kubisch. - Die Cylinderzellen haben die Höhe von etwa 0,024.

Dieser äusserst frappante Befund forderte natürlich auf, jeden Fungus umbilicalis zu untersuchen, der mir seitdem unter die Hände kam. Es waren 6 an Zahl, aber jeder ohne die gewünschten Drüsen. 
Fünf waren nichts als reine Granulome ohne Epithelbekleidung, der eine war zur Hälfte von Epithel überzogen: deutliche sehr schmale Hornschicht, deutliches Rete Malpighii mit schönen Riffzellen; letzteres sendet zahlreiche solide Zapfen in das zellenreiche Granulationsgewebe, Papillarbildungen zwischen denselben lassend.

Während nun diese Epithelbekleidung entschieden dem Hornblatte entstammte, so war es natürlich für die des erstbeschriebenen Tumors mehr als zweifelhaft. Ein ganz heterogenes Epithel - kubisches, einschichtiges - Drüs en von oben bis unten mit Cylinderzellen ausgekleidet, konnten schwerlich von der benachbarten Haut des Nabelringes über den Fungus gewachsen sein, die mussten anders woher stammen, und es lag also die Vermuthung sehr nahe, dass ihnen eins der beiden Gebilde des Nabelstranges, von denen bis dahin bekannt war, dass sie bis zu irgend welcher Fötalperiode mit Epithel ansgekleidet sind, Ductus omphalo-entericus oder Allantois zum Mutterboden gedient hatte. Auch ich machte damals Schnitte durch Nabelschnüre und fand einen epitheltragenden Hohlraum, den ich von vornherein für den Rest der Allantois hielt - nicht für den des Ductus omphalo entericus - da ich ihn an frühgeborenen Kindern (6monatlich) in fortlaufenden über den Nabelring hinausgehenden Querschnitten bis in den Urachus verfolgen konnte.

Seit Beginn des Winters ist mir kein Fungus umbilicalis wieder zu Gesicht gekommen - hohe Aussentemperatur scheint das Entstehen dieser kleinen Tumoren zu begünstigen - und ich würde jetzt von Neuem diese Untersuchungen fortgesetzt haben, nux um noch ein solches Adenom zu finden und diese Beobachtung nicht als vereinzelt dastehen $\mathrm{zu}$ lassen.

Das, was mich zur Veröffentlichung dieser einzelnen Beobachtung veranlasst, sind die Publicationen von Ahlfeld, Zini, Ruge und Sabine über den vierten Kanal im Nabelstrange. Da die von mir gefundenen Drüsen nicht als Abkömmlinge des Epidermoidalblattes anzusehen sind, so ist es zwingend, in ihnen Derivate des Epithels dieses vierten Kanales, des Restes der Allantois, zu erkennen.

Halle, 28. März 1875. 